\title{
On weak solutions of the boundary value problem within linear dilatational strain gradient elasticity for polyhedral Lipschitz domains
}

\author{
Victor A. Eremeyev \\ DICAAR, University of Cagliari, Italy Gdańsk University of Technology, Poland \\ Institute of Applied Mechanics, Russian Academy of Sciences, Russia
}

Francesco dell'Isola

Institute of Applied Mechanics, Russian Academy of Sciences, Russia

DICEAA and International Research Center on Mathematics and

Mechanics of Complex System (M\&MOCS), Università dell'Aquila, Italy

\begin{abstract}
We provide the proof of an existence and uniqueness theorem for weak solutions of the equilibrium problem in linear dilatational strain gradient elasticity for bodies occupying, in the reference configuration, Lipschitz domains with edges. The considered elastic model belongs to the class of so-called incomplete strain gradient continua whose potential energy density depends quadratically on linear strains and on the gradient of dilatation only. Such a model has many applications, e.g., to describe phenomena of interest in poroelasticity or in some situations where media with scalar microstructure are necessary. We present an extension of the previous results by Eremeyev et al. (2020 Z angew Math Phys 7l(6): $\mathrm{I}-16)$ to the case of domains with edges and when external line forces are applied. Let us note that the interest paid to Lipschitz polyhedra-type domains is at least twofold. First, it is known that geometrical singularity of the boundary may essentially influence singularity of solutions. On the other hand, the analysis of weak solutions in polyhedral domains is of great significance for design of optimal computations using a finite-element method and for the analysis of convergence of numerical solutions.
\end{abstract}

\section{Keywords}

weak solution, dilatational strain gradient elasticity, Sobolev spaces, Lipschitz polyhedra, edge forces, $H(\operatorname{div}, V)$

\section{Introduction}

Recent advances in material engineering and nanotechnologies have resulted in a growing interest in the search for generalized continuum models. Among these models the strain gradient (or, when referring to displacement field, second gradient elasticity) found various applications to the modeling of material behavior at small scales [1-3] as well as to the description of composite materials showing large variations in material properties at 
the micro-level [4-6]. In addition the theory of metamaterials and the problem of design and synthesis of metamaterials [7] has exploited the results previously obtained in the field of generalized continua.

Exotic mechanical behavior is demanded to metamaterials and second gradient continua exhibit several interesting exotic mechanical behaviors. It has to be remarked that particularly exotic is the behavior of those second gradient continua in which the deformation energy volume density does not depend on all the secondorder partial derivatives of the displacement. In such cases, one can observe (and such a phenomenology can be exploited in applications) the onset of so-called floppy modes. These modes are homogeneous deformations that are related to vanishing (or very small) deformation energy: they share this property with rigid motions.

Therefore, when non-rigid floppy modes are possible in some materials many unexpected and non-standard phenomena may occur. In the theory of metamaterials the existence of such kind of non-standard behavior is designed willingly in order to exploit it in materials engineering (see, e.g., [7-9]). An interesting example of the possible uses of incomplete second gradient materials was given in [10], where it was proposed to exploit the existence of floppy modes in order to design metamaterials that are capable of large deformations while remaining in the elastic regime. Of course, the ways in which a material showing floppy modes must be "clamped" in order to be "blocked" in such a way that equilibrium can be attained need to be determined via a suitable analysis, which has been presented, for the specific metamaterial considered there, in [11].

Toupin-Mindlin strain gradient elasticity, introduced in [12-15], is based on the introduction of a potential energy density which depends on the first and second gradient of displacement. When considering the associated formulation of the virtual work principle (VWP) it is obvious that these first and second gradients are "dual in work" to some tensor quantities which can be called stresses and hyper-stresses (double, triple, and higher-order stress tensors).

Various simplified versions of Toupin-Mindlin general model were presented in the literature (see, e.g., [16-19] and the reference therein). In particular, recently, the new model was considered [20-22]. Within this model the deformation energy volume density depends on strains $\varepsilon$ and on the gradient of dilatation $\nabla \operatorname{tr} \varepsilon$ only. The model is aimed, for example, to describe the phenomenology involved in poroelasticity [23, 24] or in those situations where microstretch elasticity [25] or continua with microstructure are required. The relations between dilatational strain gradient elasticity with the Cowin-Nunziato poroelastic model $[23,24]$ or with the Korteweg-Cahn-Hilliard capillary uids [26,27,27-29] have been fully discussed in [21] to which we refer. From the mathematical point of view the system of partial differential equations (PDEs), which describes the equilibrium in dilatational gradient elasticity does constitute a particular boundary-value problem which is not strongly elliptic in sense of $[30,31]$. As a consequence one is led to the necessity of suitably modifying the mathematical proof of the well-posedness of the corresponding equilibrium problem.

In fact, also in the present case, both the demonstration of well-posedness results and the efficient numerical solution of systems of PDEs (as very often happens in mathematical physics and mechanics) must be obtained by using the techniques based on the VWP. Among the techniques used to obtain numerical solutions we mention here finite-element, finite-difference, Galerkin and Ritz methods.

The solutions that verify a form of VWP are usually called weak solutions. In the case of generalized continua including strain gradient continua the VWP was discussed in many works, see landscape papers by Germain [32, 33], Sedov [34-36], and discussions in [37, 38]. For more recent applications of the VWP in strain gradient media we refer to [39-43] and the reference therein

Usually smoothness properties of weak solutions can be characterized when recognizing that they must belong to suitably defined Sobolev's spaces (see, e.g., [44-48]). On the other hand, geometrical singularities of domains may significantly change the properties of a solution in the vicinity of edges or vertices (see, e.g., $[49,50])$ : to solve this difficulty one requires specific techniques, for example, the use of weighted Sobolev's spaces [51,52]. Moreover the analysis of boundary-value problems in polygonal and polyhedral domains may also require suitable extensions of trace theorems, i.e. properties of restriction of weak solutions, as elements of suitable Sobolev's spaces, on a boundary with edges, see [53-55].

Similar to the Kirchhoff plate theory, the Toupin-Mindlin strain gradient elasticity [12-15] results in a system of PDEs of fourth order. The corresponding weak solutions are defined in Sobolev's space $W^{2,2}$, see [56] and the next section for definitions. Under coercivity and ellipticity weak solutions of such PDEs were studied in many works (see, e.g., [30, 31, 44-46, 48, 57-59]). Nevertheless, when strain gradient models are gradient incomplete, as happens in the case of dilatational strain gradient elasticity, the general theory based on $W^{2,2}$ space has to be modified through the most appropriate choice of that functional space where the coercivity of considered deformation energy is still valid. This situation is not completely new, as the deformation energy density (and the corresponding systems of equations) proposed for pantographic structures $[7-9,60]$ produces 
also a gradient incomplete model: therefore, for the analysis of the related boundary-value problems, a suitable anisotropic Sobolev's space had to be used in [11, 61, 62]. In fact, another example of the gradient incomplete model is given by the dilatational strain gradient elasticity as it requires, for formulating well-posedness results, the introduction of a specific generalization of $H(\operatorname{div}, V)$ space (see [20]). Roughly speaking one can say that, in incomplete strain gradient continua (i.e. in continua whose deformation energy does not depend on all second partial derivatives of the displacement field), an apparent lack of coercivity is observed, if one chooses the usual energy space. In fact, such an apparent lack of coercivity is overcome by choosing the appropriate anisotropic Sobolev space as energy space. In such space the incomplete energy results indeed to be coercive, and therefore the standard scheme of proof of well-posedness can be adapted. Obviously the set of existing traces of the functions belonging to the appropriate anisotropic Sobolev spaces has to be clearly specified, also in order to establish the appropriate class of essential boundary conditions that can be assigned in considered equilibrium problems.

The aim of the present paper is to extend the recent analysis already provided in [20] for the dilatational strain gradient elasticity to the case of polyhedral Lipschitz domains. In other words, we wanted to consider here domains that are only piecewise smooth, as their boundary is allowed to contain a finite number of edges and vertices. The interest in considering such class of "irregular" is manifold. They may appear as a result of design, for example, when a domain is a union or intersection of some primitive shapes as cylinders, plates, balls, etc. These boundaries maybe also related to the singularities appearing in presence of cracks, or of abrupt changes of material properties. However, it is also worth mentioning that polygonal (in $\mathbb{R}^{2}$ ) and polyhedral (in $\mathbb{R}^{3}$ ) approximations of domains are widely used for calculations of numerical solutions by means of the finiteelement method. In this case edges and vertices appear artificially as a result of the approximation technique. Nevertheless, independently on the nature of edges, such singularities may essentially influence an accuracy and convergence of numerical methods.

The paper is organized as follows. In Section 2, we briefly discuss the necessary requirements to the geometry of domains and introduce related Sobolev's spaces. In Section 3, we present the constitutive relations of the dilatational strain gradient elasticity. Using the VWP in Section 4, we derive equilibrium conditions. The particular attention is paid to edges and to the compatibility conditions along edges. In addition, we consider external forces acting along surface curves. Finally, in Section 5, we formulate requirements to external forces and double forces under which we prove existence and uniqueness of weak solutions. In the following, we used direct (index-free) tensor analysis, so vectors, second- and higher-order tensors will be denoted by boldface symbols as in $[42,63]$.

\section{Preliminaries}

Let $V$ be an open bounded polyhedral domain in $\mathbb{R}^{3}$ with a piecewise smooth Lipschitz boundary $S=\partial V$ (see, e.g., [51, 64] for more details). Here $S$ consists of $C^{1}$-faces $S_{i}, i=1, \ldots, N, C^{1}$-edges $L_{i j}=\bar{S}_{i} \cap \bar{S}_{j}$, and vertices, see Figure 1 where three faces $S_{1}, S_{2}, S_{3}$ and three edges $L_{12}, L_{13}, L_{23}$ are shown. Here $\mathbf{n}$ is a unit vector of an outward normal to $S$. We also assume that contact angles between neighboring faces is not zero nor $2 \pi$. Thus, the boundary satisfies the regularity requirements of Sobolev's embedding theorems $[44,48,49,56]$.

In the following, we use standard spaces of continuous functions $C^{m}$, Lebesgue $L^{p}$, and Sobolev's $W^{m, p}$ and $W_{0}^{m, p}$ spaces. In particular, we consider $W^{1,2}(V)$ and $W^{2,2}(V)$ equipped with the norms

$$
\begin{aligned}
\|u\|_{W^{1,2}(V)} & =\left[\iiint_{V}\left[|u|^{2}+\nabla u \cdot \nabla u\right] d V\right]^{1 / 2}, \\
\|u\|_{W^{2,2}(V)} & =\left[\iiint_{V}\left[|u|^{2}+\nabla u \cdot \nabla u+\nabla \nabla u: \nabla \nabla u\right] d V\right]^{1 / 2},
\end{aligned}
$$

where $\nabla$ is the three-dimensional (3D) nabla operator. Hereinafter "." and "." denote the dot product and the inner product in the space of second-order tensors. For example, for vectors and dyads we have [42, 65]

$$
\begin{gathered}
\mathbf{a} \cdot \mathbf{b}=a_{i} b_{i}, \quad(\mathbf{a} \otimes \mathbf{b}) \cdot \mathbf{c}=(\mathbf{b} \cdot \mathbf{c}) \mathbf{a}, \\
\mathbf{a} \cdot(\mathbf{b} \otimes \mathbf{c})=(\mathbf{a} \cdot \mathbf{b}) \mathbf{c}, \quad(\mathbf{a} \otimes \mathbf{b}):(\mathbf{c} \otimes \mathbf{d})=(\mathbf{a} \cdot \mathbf{c})(\mathbf{b} \cdot \mathbf{d}), \ldots
\end{gathered}
$$

where " $\otimes$ " denotes the dyadic product, $a_{i}, b_{i}$ are Cartesian components of vectors $\mathbf{a}, \mathbf{b}$, respectively, and the Einstein summation rule is utilized. 


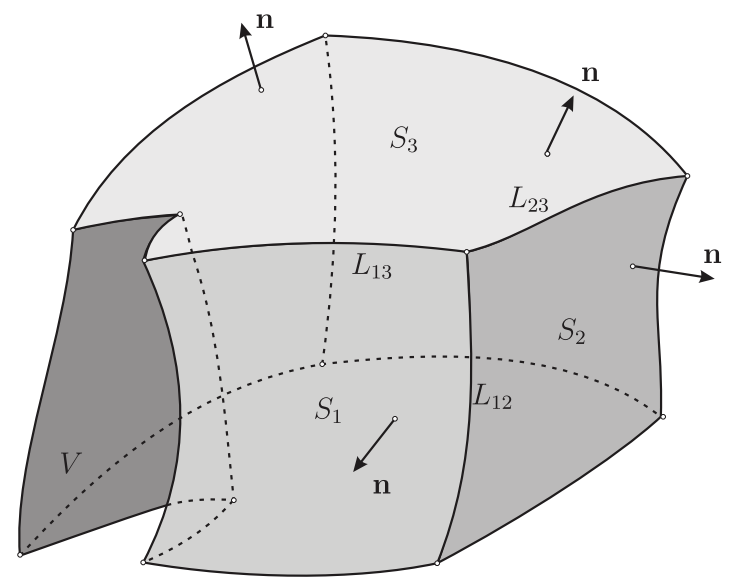

Figure I. Polyhedral Lipschitz domain $V$. Three faces and three edges are shown.

For vector-valued functions we use the notation $\mathbf{u} \in \mathbf{L}^{P}$ or $\mathbf{u} \in \mathbf{W}^{m, p}$, etc., if any Cartesian component of $\mathbf{u}$ belongs to $L^{p}$ or to $W^{m, p}$.

In addition we introduce the functional spaces $H(\operatorname{div}, V)$ (see, e.g., $[47,66])$ and $\mathbf{H}_{1}(\operatorname{div}, V)$ [20]

$$
\begin{aligned}
H(\operatorname{div}, V) & =\left\{\mathbf{u}: \mathbf{u} \in \mathbf{L}^{2}(V), \nabla \cdot \mathbf{u} \in L^{2}(V)\right\}, \\
\mathbf{H}_{1}(\operatorname{div}, V) & =\left\{\mathbf{u}: \mathbf{u} \in \mathbf{W}^{1,2}(V), \nabla \nabla \cdot \mathbf{u} \in \mathbf{L}^{2}(V)\right\} .
\end{aligned}
$$

The spaces $H(\operatorname{div}, V)$ and $\mathbf{H}_{1}(\operatorname{div}, V)$ are Hilbert spaces with inner products

$$
\begin{aligned}
(\mathbf{u}, \mathbf{v})_{H(\operatorname{div}, V)} & =\iiint_{V}[\mathbf{u} \cdot \mathbf{v}+(\nabla \cdot \mathbf{u})(\nabla \cdot \mathbf{v})] d V \\
(\mathbf{u}, \mathbf{v})_{\mathbf{H}_{1}(\operatorname{div}, V)} & =\iiint_{V}[\mathbf{u} \cdot \mathbf{v}+\nabla \mathbf{u}: \nabla \mathbf{v}+(\nabla \nabla \cdot \mathbf{u}) \cdot(\nabla \nabla \cdot \mathbf{v})] d V .
\end{aligned}
$$

The corresponding norms are

$$
\|\mathbf{u}\|_{H(\operatorname{div}, V)}=(\mathbf{u}, \mathbf{u})_{H(\operatorname{div}, V)}^{1 / 2}, \quad\|\mathbf{u}\|_{\mathbf{H}_{1}(\operatorname{div}, V)}=(\mathbf{u}, \mathbf{u})_{\mathbf{H}_{1}(\operatorname{div}, V)}^{1 / 2},
$$

respectively.

Together with the functional space $H(\operatorname{curl}, V)$, also the space $H(\operatorname{div}, V)$ found applications in some problems of mathematical physics such as hydrodynamics or Maxwell's equations [47,64,67]. As was shown in [20], $\mathbf{H}_{1}(\operatorname{div}, V)$ is a natural (energy) functional space for static boundary-value problems within the dilatational strain gradient elasticity. The related properties of $\nabla$ div operator were studied in $[68,69]$.

\section{Potential energy density}

Let us consider small deformations of an elastic solid body $\mathcal{B}$ which occupies a domain $V$ under various external loadings which include forces and double forces distributed in $V$ and on its boundary $S=\partial V$. In the dilatational strain gradient elasticity a potential energy density $W$ is introduced as a function of linear strains and the gradient of dilatation

$$
W=W(\varepsilon, \nabla \epsilon), \quad \boldsymbol{\varepsilon}=\boldsymbol{\varepsilon}(\mathbf{u}) \equiv \frac{1}{2}\left(\nabla \mathbf{u}+\nabla \mathbf{u}^{\mathrm{T}}\right), \quad \epsilon=\epsilon(\mathbf{u}) \equiv \operatorname{tr} \boldsymbol{\varepsilon}(\mathbf{u}),
$$

where $\mathbf{u}=\mathbf{u}(\mathbf{x})$ is a displacement vector and superscript $\mathrm{T}$ denotes transpose of a second-order tensor. For small deformations we assume that $W$ is a quadratic form of $\varepsilon$ and $\mathbf{k}=\nabla \epsilon$, so it takes the form

$$
W=W(\boldsymbol{\varepsilon}, \mathbf{k})=\frac{1}{2} \boldsymbol{\varepsilon}: \mathbf{C}: \boldsymbol{\varepsilon}+\boldsymbol{\varepsilon}: \mathbf{B} \cdot \mathbf{k}+\frac{1}{2} \mathbf{k} \cdot \mathbf{D} \cdot \mathbf{k}
$$


where $\mathbf{C}$ is a fourth-order tensor of elastic moduli defined as in linear elasticity [63], $\mathbf{B}$ is a third-order coupling tensor, it has symmetry properties similar to flexoelectric tensors [70, 71], and $\mathbf{D}$ is a second-order symmetric tensor related to the gradient of dilatation. For an isotropic material $W$ takes the form

$$
W=\frac{1}{2} \lambda \epsilon^{2}+\mu \varepsilon: \boldsymbol{\varepsilon}+\frac{1}{2} \alpha \mathbf{k} \cdot \mathbf{k}
$$

with two Lamé elastic moduli $\lambda$ and $\mu$, and a dilatational gradient modulus $\alpha>0$.

In the following, we require that $W$ be a positive-definite function, so we have the inequality

$$
W(\boldsymbol{\varepsilon}, \mathbf{k}) \geq C_{1} \boldsymbol{\varepsilon}: \boldsymbol{\varepsilon}+C_{2} \mathbf{k} \cdot \mathbf{k}, \quad C_{1}, C_{2}>0,
$$

where $C_{1}$ and $C_{2}$ are positive constants independent on $\varepsilon$ and $\mathbf{k}$.

\section{VWP}

The static boundary-value problem can be derived from the stationarity condition of a total energy functional, i.e. using the Lagrange variational principle extended for strain gradient elasticity [39-42]. The total energy functional takes the form

$$
E[\mathbf{u}]=\iiint_{V} W d V-F[\mathbf{u}],
$$

where $F[\mathbf{u}]$ is a linear functional which describes the work of external loadings. We specify the specific form of $F[\mathbf{u}]$ after the analysis of the first variation of the potential energy functional

$$
U[\mathbf{u}]=\iiint_{V} W d V .
$$

Similarly to what was done in [20], first we obtain

$$
\delta U=\delta U[\mathbf{u}, \mathbf{v}]=\iiint_{V}[\boldsymbol{\sigma}(\mathbf{u}): \boldsymbol{\varepsilon}(\mathbf{v})+\mathbf{m}(\mathbf{u}) \cdot \mathbf{k}(\mathbf{v})] d V,
$$

where $\mathbf{v}$ is a variation of $\mathbf{u}, \mathbf{v}=\delta \mathbf{u}$, and we have introduced the stress tensor $\sigma$ and the double force vector $\mathbf{m}$ by the formulas

$$
\begin{aligned}
\boldsymbol{\sigma} & \equiv \frac{\partial W}{\partial \boldsymbol{\varepsilon}}=\mathbf{C}: \boldsymbol{\varepsilon}+\mathbf{B} \cdot \mathbf{k}, \\
\mathbf{m} & \equiv \frac{\partial W}{\partial \mathbf{k}}=\boldsymbol{\varepsilon}: \mathbf{B}+\mathbf{D} \cdot \mathbf{k} .
\end{aligned}
$$

In the following, we assume that a face or an union of faces $S_{u}$ of $S$ is clamped, in Figure 2 this area is shown as shaded by lines. Thus, we obtain the kinematic boundary conditions

$$
\left.\mathbf{u}\right|_{S_{u}}=\mathbf{0},\left.\quad \mathbf{n} \cdot \frac{\partial \mathbf{u}}{\partial n} \equiv \frac{\partial u_{n}}{\partial n}\right|_{S_{u}}=0,
$$

where $\mathbf{n}$ is a unit vector of outward normal to $S$ and $\partial / \partial n=\mathbf{n} \cdot \nabla$ denotes the normal derivative. As $\mathbf{v}$ is the variation of $\mathbf{u}, \mathbf{v}$ also satisfies (12).

Integrating by parts in (9), we obtain

$$
\begin{aligned}
\delta U= & -\iiint_{V}[(\nabla \cdot \boldsymbol{\sigma}(\mathbf{u})) \cdot \mathbf{v}+(\nabla \cdot \mathbf{m}(\mathbf{u})) \nabla \cdot \mathbf{v}] d V \\
& +\iint_{S_{t}}[\mathbf{n} \cdot \boldsymbol{\sigma}(\mathbf{u}) \cdot \mathbf{v}+(\mathbf{n} \cdot \mathbf{m}(\mathbf{u})) \nabla \cdot \mathbf{v}] d S \\
= & \iiint_{V}[-\nabla \cdot \boldsymbol{\sigma}(\mathbf{u})+\nabla(\nabla \cdot \mathbf{m}(\mathbf{u}))] \cdot \mathbf{v} d V \\
& +\iint_{S_{t}}[\mathbf{n} \cdot \boldsymbol{\sigma}(\mathbf{u}) \cdot \mathbf{v}-(\nabla \cdot \mathbf{m}(\mathbf{u})) \mathbf{n} \cdot \mathbf{v}+(\mathbf{n} \cdot \mathbf{m}(\mathbf{u})) \nabla \cdot \mathbf{v}] d S .
\end{aligned}
$$


In (13) $S_{t}=S \backslash S_{u}$ and we have taken into account (12). Further transformations of $\delta U$ require integration by parts over the boundary as in [20,40]. Introducing the surface nabla operator by

$$
\nabla_{s}=(\mathbf{I}-\mathbf{n} \otimes \mathbf{n}) \cdot \nabla
$$

we obtain

$$
\nabla \cdot \mathbf{v}=\mathbf{n} \cdot \frac{\partial \mathbf{v}}{\partial n}+\nabla_{s} \cdot \mathbf{v}
$$

where $\mathbf{I}$ is the $3 \mathrm{D}$ unit tensor. For any face $S_{i} \subset S_{t}$ we have the formula

$$
\begin{aligned}
\iint_{S_{i}}(\mathbf{n} \cdot \mathbf{m}(\mathbf{u})) \nabla_{s} \cdot \mathbf{v} d S= & \iint_{S_{i}}\left\{\nabla_{s} \cdot[(\mathbf{n} \cdot \mathbf{m}) \mathbf{v}]-\nabla_{S}(\mathbf{n} \cdot \mathbf{m}) \cdot \mathbf{v}\right\} d S \\
= & \iint_{S_{i}}\left[-2 H(\mathbf{n} \cdot \mathbf{m})(\mathbf{n} \cdot \mathbf{v})-\nabla_{S}(\mathbf{n} \cdot \mathbf{m}) \cdot \mathbf{v}\right] d S \\
& +\int_{\partial S_{i}}(\mathbf{n} \cdot \mathbf{m}) \mathbf{v} \cdot \mathbf{v} d s,
\end{aligned}
$$

where $H=-1 / 2 \nabla_{S} \cdot \mathbf{n}$ is the mean curvature of $S_{i}, \boldsymbol{v}=\boldsymbol{v}_{i}$ is a unit vector of outer normal to $\partial S_{i}$ such that $\boldsymbol{v} \cdot \mathbf{n}=0$. Using (9) and (14) we transform $\delta U$ into

$$
\begin{aligned}
\delta U= & \iiint_{V}[-\nabla \cdot \boldsymbol{\sigma}+\nabla(\nabla \cdot \mathbf{m})] \cdot \mathbf{v} d V \\
& +\iint_{S_{t}}\left[\mathbf{n} \cdot \boldsymbol{\sigma}-(\nabla \cdot \mathbf{m}) \mathbf{n}-2 H(\mathbf{n} \cdot \mathbf{m}) \mathbf{n}-\nabla_{s}(\mathbf{n} \cdot \mathbf{m})\right] \cdot \mathbf{v} d S . \\
& +\iint_{S_{t}}(\mathbf{n} \cdot \mathbf{m}) \mathbf{n} \cdot \frac{\partial \mathbf{v}}{\partial n} d S \\
& +\sum_{i, j: L_{i j} \subset \bar{S}_{t}} \int_{L_{i j}} \llbracket(\mathbf{n} \cdot \mathbf{m}) \boldsymbol{v} \rrbracket_{i j} \cdot \mathbf{v} d s .
\end{aligned}
$$

Here the double square brackets denote a sum $\llbracket(\ldots) \rrbracket_{i j}=(\ldots)_{i}+(\ldots)_{j}$, thus,

$$
\llbracket(\mathbf{n} \cdot \mathbf{m}) \boldsymbol{v} \rrbracket_{i j}=\left(\mathbf{n}_{i} \cdot \mathbf{m}_{i}\right) \boldsymbol{v}_{i}+\left(\mathbf{n}_{j} \cdot \mathbf{m}_{j}\right) \boldsymbol{v}_{j}
$$

for faces $S_{i}$ and $S_{j}$ having a common edge $L_{i j}$.

Obviously, $\delta U$ is a sum of volume, surface, and linear integrals linearly dependent on $\mathbf{v}$ and $\mathbf{n} \cdot \partial \mathbf{v} / \partial n$. Thus, a compatible form of $F(\mathbf{v})$ may also contain the same integrals. As a result, we consider first $F(\mathbf{v})$ in the following form

$$
F(\mathbf{v})=\iiint_{V} \mathbf{f} \cdot \mathbf{v} d V+\iint_{S_{t}}\left(\mathbf{t} \cdot \mathbf{v}+c \mathbf{n} \cdot \frac{\partial \mathbf{v}}{\partial n}\right) d S+\int_{\Lambda} \mathbf{g} \cdot \mathbf{v} d s,
$$

where $\mathbf{f}$ is a vector-valued field of volumetric forces, $\mathbf{t}$ is a vector-valued field of surface traction, $c$ is a scalar surface field of double force, and $\mathbf{g}$ is a vector-valued field of line forces, i.e. forces acting on some surface curves. For brevity, we use the notation $\Lambda=\bigcup_{i, j} L_{i j} \cup L, L_{i j} \subset \bar{S}_{t}$, where $L$ is an union of surface $C^{1}$-curves where line forces are prescribed. We assumed that $L$ consists of closed surface smooth curves or smooth curves which end on edges. Thus, if a line force is prescribed on face $S_{i}$, it can be also considered as a finite union of parts $S_{i}^{k}, S_{i}=\bigcup_{k} S_{i}^{k}$, whereas $L$ with edges constitute boundaries of sub-faces $S_{i}^{k}$. For example, in Figure 2 we have introduced three parts of $S_{3}, S_{3}=S_{3}^{1} \cup S_{3}^{2} \cup S_{3}^{3}$.

As a result, from

$$
\delta U[\mathbf{u}, \mathbf{v}]-F(\mathbf{v})=0,
$$

we obtain the equilibrium equations and the natural boundary conditions

$$
\begin{array}{ll}
\mathbf{x} \in V: & \nabla \cdot \boldsymbol{\sigma}-\nabla(\nabla \cdot \mathbf{m})+\mathbf{f}=\mathbf{0}, \\
\mathbf{x} \in S_{t}: & \mathbf{n} \cdot \boldsymbol{\sigma}-(\nabla \cdot \mathbf{m}) \mathbf{n}-2 H(\mathbf{n} \cdot \mathbf{m}) \mathbf{n}-\nabla_{s}(\mathbf{n} \cdot \mathbf{m})=\mathbf{t}, \\
& \mathbf{n} \cdot \mathbf{m}=c, \\
\mathbf{x} \in \Lambda: & \llbracket(\mathbf{n} \cdot \mathbf{m}) \boldsymbol{v} \rrbracket_{\Lambda}=\mathbf{g} .
\end{array}
$$




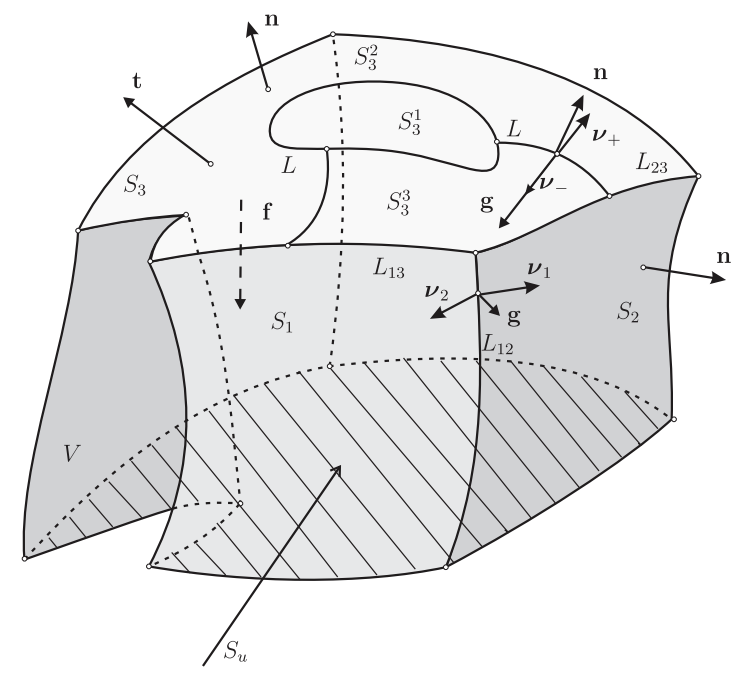

Figure 2. Polyhedral Lipschitz domain $V$. On face $S_{3}$ three sub-faces $S_{3}^{1}, S_{3}^{2}, S_{3}^{3}$ are shown, which are separated by curve $L$. External vector-valued fields $\mathbf{f}, \mathbf{t}$, and $\mathbf{g}$ are also shown schematically.

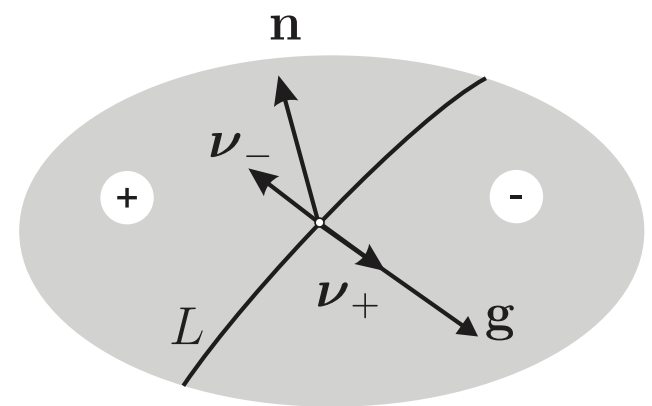

Figure 3. Interface curve on a smooth surface.

Note, that using (19) we can simplify (18) and (20) as follows

$$
\begin{array}{ll}
\mathbf{x} \in S_{t}: & \mathbf{n} \cdot \boldsymbol{\sigma}-(\nabla \cdot \mathbf{m}) \mathbf{n}-2 H c \mathbf{n}-\nabla_{s} c=\mathbf{t}, \\
\mathbf{x} \in \Lambda: & \llbracket c \boldsymbol{v} \rrbracket_{\Lambda}=\mathbf{g} .
\end{array}
$$

Equation (21) shows that a surface double force contributes into a surface traction. Indeed, instead of $\mathbf{t}$ we can consider $\tilde{\mathbf{t}}=\mathbf{t}+2 H c \mathbf{n}+\nabla_{s} c$ as an external load.

Equation (22) is a static compatibility condition which relates surface double force with line forces. For an edge, we have a jump in $\boldsymbol{v}: \llbracket v \rrbracket_{L_{i j}} \neq \mathbf{0}$, so for $c \neq 0$ we have to apply a line force $\mathbf{g}$ in order to have a mechanical equilibrium. In other words, for non-trivial double forces we need to introduce line forces acting on edges, see also $[72,73]$. On the other hand, for a smooth face $(22)$ takes the form

$$
\llbracket c \boldsymbol{v} \rrbracket_{L} \equiv c_{+} \boldsymbol{v}_{+}+c_{-} \boldsymbol{v}_{-}=\mathbf{g},
$$

where \pm denotes unilateral limits on $L$, see Figure 3. As here $\boldsymbol{v}_{+}=\boldsymbol{v}=-\boldsymbol{v}_{-}$, Equation (22) transforms into

$$
\left(c_{+}-c_{-}\right) \boldsymbol{v}=\mathbf{g} .
$$

Thus, $\mathbf{g}$ should be collinear to $\boldsymbol{v}, \mathbf{g}=G \mathbf{v}$, and $G=c_{+}-c_{-}$. In other words, for a smooth surface a line force is possible only if it lies in the tangent plane to the surface and it should be related to a jump of surface double forces.

From the computational point of view this analysis seems to be rather important. Indeed, polygonal and polyhedral approximations of an initially smooth surface are widely applied using a finite-element analysis (FEA). In order to satisfy the mechanical equilibrium one has to apply fictive edge forces to such artificially appearing edges as finite-element boundaries, and these edge forces should be consistent with (22). 


\section{Weak solutions: existence and uniqueness}

In order to analyze the well-posedness of the problem under consideration we shall prove existence and uniqueness of its weak solution. To this end we use the same technique as applied in [20]. First, we introduce a functional energy space as follows.

Definition 5.1. The energy space $\mathbf{E}$ is the completion of $\mathbf{C}^{2}(V)$ vector-valued functions satisfying boundary conditions (12) in the norm

$$
\|\mathbf{u}\|_{e}=\left[\iiint_{V}[\sigma(\mathbf{u}): \boldsymbol{\varepsilon}(\mathbf{u})+\mathbf{m}(\mathbf{u}) \cdot \mathbf{u}(\mathbf{u})] d V\right]^{1 / 2},
$$

where $\boldsymbol{\sigma}$ and $\mathbf{m}$ are given by (10) and (11), respectively.

Note that in the following we treat all quantities as dimensionless. One can prove that $\|\cdot\|_{e}$ has all properties of the norm. Moreover, $\mathbf{E}$ is a separable Hilbert space with the inner product defined by

$$
(\mathbf{u}, \mathbf{v})_{e}=\iiint_{V}[\boldsymbol{\sigma}(\mathbf{u}): \boldsymbol{\varepsilon}(\mathbf{v})+\mathbf{m}(\mathbf{u}) \cdot \mathbf{k}(\mathbf{v})] d V .
$$

Using (6), we see that the norm in $\mathbf{E}$ is equivalent to the norm in $\mathbf{H}_{1}(\operatorname{div}, V)$, i.e. for $\mathbf{u} \in \mathbf{E}$ we have inequalities

$$
C_{3}\|\mathbf{u}\|_{\mathbf{H}_{1}(\operatorname{div}, V)} \leq\|\mathbf{u}\|_{e} \leq C_{4}\|\mathbf{u}\|_{\mathbf{H}_{1}(\operatorname{div}, V)},
$$

where $C_{3}$ and $C_{4}$ are positive constants independent on $\mathbf{u}$.

Let us consider the decomposition of $F(\mathbf{v})$

$$
F(\mathbf{v})=F_{1}(\mathbf{v})+F_{2}(\mathbf{v}),
$$

where

$$
\begin{aligned}
& F_{1}(\mathbf{v})=\iiint_{V} \mathbf{f} \cdot \mathbf{v} d V+\iint_{S_{t}} \mathbf{t} \cdot \mathbf{v} d S \\
& F_{2}(\mathbf{v})=\iint_{S_{t}} c \mathbf{n} \cdot \frac{\partial \mathbf{v}}{\partial n} d S+\int_{\Lambda} \mathbf{g} \cdot \mathbf{v} d s
\end{aligned}
$$

$F_{1}(\mathbf{v})$ is a linear functional which appears in linear elasticity (see, e.g., $\left.[42,45,48,74]\right)$, whereas $F_{2}(\mathbf{v})$ relates to surface double forces. As we mentioned previously, line forces appear on edges as a result of static balance of surface double forces, and on surface curves where $c$ undergoes a discontinuity jump. Replacing $\mathbf{g}$ using (22) we transform $F_{2}(\mathbf{v})$ into the form

$$
F_{2}(\mathbf{v})=\iint_{S_{t}} c \mathbf{n} \cdot \frac{\partial \mathbf{v}}{\partial n} d S+\int_{\Lambda} \llbracket c \mathbf{v} \rrbracket_{\Lambda} \cdot \mathbf{v} d s
$$

As $F_{2}(\mathbf{v})$ describes the virtual work related to surface double forces, we see that the virtual work also depends on discontinuity jumps of $c$.

Using the introduced energy space we now define a weak solution as follows.

Definition 5.2. We call $\mathbf{u}_{0} \in \mathbf{E}$ a weak solution of the boundary value problem (17)-(20) and (12) if it satisfies the relation

$$
\left(\mathbf{u}_{0}, \mathbf{v}\right)_{E}=F_{1}(\mathbf{v})+F_{2}(\mathbf{v}) \quad \forall \mathbf{v} \in \mathbf{E} .
$$

Now the well-posedness of the problem under consideration is given by the following theorem.

Theorem 5.3. Let $V \subset \mathbb{R}^{3}$ be a bounded polyhedral Lipschitz domain with $C^{1}$ faces, $\mathbf{f} \in \mathbf{L}^{6 / 5}(V)$, $\mathbf{t} \in \mathbf{L}^{4 / 3}\left(S_{1}\right)$, and $c \in \prod_{i, k} W^{1,4 / 3}\left(S_{i}^{k}\right), S_{i}^{k} \subset \bar{S}_{t}$. Then there exists a weak solution $\mathbf{u}_{0} \in \mathbf{E}$. It is unique. Furthermore,

$$
J\left(\mathbf{u}_{0}\right)=\inf _{\mathbf{u} \in E} J(\mathbf{u})
$$


where

is the total energy functional.

$$
J(\mathbf{u})=\frac{1}{2}(\mathbf{u}, \mathbf{u})_{e}-F_{1}(\mathbf{u})-F_{2}(\mathbf{u})
$$

Let us note that unlike [20], where we required that $c \in W^{1,2}\left(S_{t}\right)$, here we relax this requirement considering piecewise differentiable functions. The used notation $c \in \prod_{i, k} W^{1,4 / 3}\left(S_{i}^{k}\right)$ means that a restriction of $c$ on $S_{i}^{k}$ belongs to $W^{1,4 / 3}\left(S_{i}^{k}\right):\left.c\right|_{S_{i}^{k}} \in W^{1,4 / 3}\left(S_{i}^{k}\right)$.

Proof. As $\mathbf{E} \subset \mathbf{W}^{1,2}(V)$, under required conditions on $\mathbf{f}$ and $\mathbf{t}, F_{1}(\mathbf{v})$ is a linear continuous functional in $\mathbf{E}$ (see, e.g., $[20,74])$.

In order to prove the boundedness of $F_{2}(\mathbf{v})$ we shall modify the proof given in [20] as follows. As $\mathbf{C}^{2}(V)$ is dense in $\mathbf{E}$, we consider $\mathbf{v} \in \mathbf{C}^{2}(V)$. Using the decomposition on any face

$$
\epsilon(\mathbf{v}) \equiv \nabla \cdot \mathbf{v}=\nabla_{s} \cdot \mathbf{v}+\mathbf{n} \cdot \frac{\partial \mathbf{v}}{\partial n},
$$

we obtain

$$
F_{2}(\mathbf{v})=\iint_{S_{t}} c\left[\epsilon(\mathbf{v})-\nabla_{s} \cdot \mathbf{v}\right] d S+\int_{\Lambda} \llbracket c v \rrbracket_{\Lambda} \cdot \mathbf{v} d s
$$

With the surface divergence theorem [42] for any smooth sub-face we have the formula

$$
\iint_{S_{i}^{k}}\left[\nabla_{S} \cdot(c \mathbf{v})+2 H c \mathbf{n} \cdot \mathbf{v}\right] d S=\int_{\partial S_{i}^{k}} c \mathbf{v} \cdot \mathbf{v} d S .
$$

From (29) it follows the integration by parts formula

$$
\iint_{S_{i}^{k}} c\left(\nabla_{s} \cdot \mathbf{v}\right) d S=\int_{\partial S_{i}^{k}} c \mathbf{v} \cdot \mathbf{v} d S-\iint_{S_{i}^{k}}\left[\left(\nabla_{s} c\right) \cdot \mathbf{v}+2 H c \mathbf{n} \cdot \mathbf{v}\right] d S .
$$

Using (30) and taking into account that $S_{t}=\bigcup_{i, k} S_{i}^{k}$ and $\Lambda$ is a union of boundaries between faces and sub-faces, we transform $F_{2}(\mathbf{v})$ as follows

$$
F_{2}(\mathbf{v})=\sum_{i, k} \iint_{S_{i}^{k}} c \epsilon(\mathbf{v}) d S+\sum_{i, k} \iint_{S_{i}^{k}}\left[\left(\nabla_{S} c\right) \cdot \mathbf{v}+2 H c \mathbf{n} \cdot \mathbf{v}\right] d S .
$$

Note that (31) is still valid if $\mathbf{v} \in \mathbf{E}$ and $c$ satisfies the conditions of the theorem. Indeed, because $\mathbf{v} \in \mathbf{E} \subset$ $\mathbf{H}_{1}(\operatorname{div}, V), \epsilon \in W^{1,2}(V)$. Using Sobolev's embedding theorem we have $\epsilon \in L^{4}\left(S_{t}\right)$. As $\mathbf{W}^{1,2}(V) \in \mathbf{E}, \mathbf{v}$ belongs at least to $\mathbf{L}^{4}\left(S_{t}\right)$. As a result, we have the inequality

$$
\begin{aligned}
\left|F_{2}(\mathbf{v})\right| \leq & \sum_{i, k} \iint_{S_{i}^{k}}|c \epsilon(\mathbf{v})| d S+\sum_{i, k} \iint_{S_{i}^{k}}\left[\left|\left(\nabla_{S} c\right) \cdot \mathbf{v}\right|+2|H c \mathbf{n} \cdot \mathbf{v}|\right] d S \\
\leq & \sum_{i, k}\left(\iint_{S_{i}^{k}}|c|^{4 / 3} d S\right)^{3 / 4}\left(\iint_{S_{i}^{k}}|\epsilon(\mathbf{v})|^{4} d S\right)^{1 / 4} \\
& +\sum_{i, k}\left(\iint_{S_{i}^{k}}\left|\nabla_{S} c\right|^{4 / 3} d S\right)^{3 / 4}\left(\iint_{S_{i}^{k}}|\mathbf{v}|^{4} d S\right)^{1 / 4} \\
& +\sum_{i, k} 2 \max _{\mathbf{x} \in S_{i}^{k}}|H(\mathbf{x})|\left(\iint_{S_{i}^{k}}|c|^{4 / 3} d S\right)^{3 / 4}\left(\iint_{S_{i}^{k}}|\mathbf{v}|^{4} d S\right)^{1 / 4} \\
\leq & C_{5} \sum_{i, k}\left[\left(\iint_{S_{i}^{k}}|c|^{4 / 3} d S\right)^{3 / 4}+\left(\iint_{S_{i}^{k}}\left|\nabla_{s} c\right|^{4 / 3} d S\right)^{3 / 4}\right]\|\mathbf{v}\|_{e},
\end{aligned}
$$


where $C_{5}$ is a positive constant independent on $c$ and $\mathbf{v}$. If $c \in \prod_{i, k} W^{1,4 / 3}\left(S_{i}^{k}\right), F_{2}(\mathbf{v})$ is a linear continuous functional in $\mathbf{E}$.

Obviously, $F(\mathbf{v})=F_{1}(\mathbf{v})+F_{2}(\mathbf{v})$ is also linear continuous functional. From the Riesz theorem it follows that there exists an unique element $\mathbf{u}^{*} \in \mathbf{E}$ such that

$$
F(\mathbf{v})=\left(\mathbf{u}^{*}, \mathbf{v}\right)_{e}
$$

Now (27) transforms into

$$
\left(\mathbf{u}_{0}, \mathbf{v}\right)_{e}=\left(\mathbf{u}^{*}, \mathbf{v}\right)_{e} \quad \forall \mathbf{v} \in \mathbf{E} .
$$

Thus, $\mathbf{u}_{0}=\mathbf{u}^{*}$.

The minimizing property of $\mathbf{u}_{0}$ follows from the standard analysis of quadratic functionals (see, e.g., [42, 48]).

Remark. We can also extend the form of $F_{1}(\mathbf{v})$ as follows

$$
F_{1}(\mathbf{v})=\iiint_{V}\left(\mathbf{f} \cdot \mathbf{v}+\mathbf{f}_{1}: \nabla \mathbf{v}+\mathbf{f}_{2} \cdot \nabla \nabla \cdot \mathbf{v}\right) d V+\iint_{S_{t}} \mathbf{t} \cdot \mathbf{v} d S
$$

where $\mathbf{f}_{1}$ and $\mathbf{f}_{2}$ are generalized loadings related to external volumetric couples and double forces. For the boundedness of $F_{1}(\mathbf{v})$ it is sufficient to require that $\mathbf{f} \in \mathbf{L}^{6 / 5}(V), \mathbf{t} \in \mathbf{L}^{4 / 3}\left(S_{t}\right), \mathbf{f}_{1} \in \mathbf{L}^{2}(V)$, and $\mathbf{f}_{2} \in \mathbf{L}^{2}(V)$. Similar extensions of external loadings can be found in $[46,47]$.

\section{Conclusions}

In the literature, the concept of contact line force applied on the boundary of continua has been recently recognized as important when dealing with second gradient continua (see, e.g., [72,73] and references therein). Actually it has been recognized that contact interactions in second gradient continua must involve not only surface densities of forces, but also surface densities of double forces joint with line densities of forces [32,33].

In [72] the class of second gradient continua has also been characterized where it is not possible to observe line contact forces while double forces are possible. Moreover, by exploiting the concept of limit dihedral shaped contact surfaces, it is proven that it is not possible to have second gradient continua in which simultaneously edge contact line forces are present and double forces are vanishing. Such results imply that in generalized continuum mechanics the analysis of trace theorems in more complex Sobolev spaces $[49,56]$ such as weighted and anisotropic ones may play a relevant role. In fact, anisotropic Sobolev spaces supply the most suitable functional analytic structure in which the theory of incomplete second gradient linear continua can be framed: the most appropriate energy spaces for these kind of continua are exactly those spaces in which a chosen subset of second-order partial derivatives belong to the space $L^{2}$.

Of course, for functions belonging to anisotropic Sobolev spaces, not all traces on the boundaries of the considered domains do exist. As a consequence the possible essential boundary conditions that can be assigned in the equilibrium problem for incomplete second gradient can involve only those traces which actually exist. The reader will agree that, for these reasons, the careful study of the functional analytic bases of the theory of (incomplete) second gradient continua has a relevance both in the modeling process of mechanical phenomena and in the development of predictive numerical integration schemes.

In this paper, in the particular case of the linear dilatational strain gradient continuum model, the appropriate boundary value equilibrium problem has been formulated and a well-posedness result has been proven when the boundary of considered continuum, in the reference configuration, is piecewise regular (that is, has a finite number of edges concurring in a finite number of wedges) and when external forces are concentrated on a finite number of curves included on the faces of such boundary.

The studied model has deep relations to gradient-type extension of continuous models with scalar microstructure [75]: in particular, we can refer to the models studied in poroelasticity [23, 24], see also the discussion in [21].[AQ: 2]

In fact, in this paper, we have considered the particular incomplete second gradient continuum where the potential energy density depends on first gradient strain and on the gradient of the trace of the strain tensor, 
i.e. on the gradient of dilatation. From the mathematical point of view, the correspondent equilibrium system of partial differential equations is not strongly elliptic as is assumed in Toupin-Mindlin strain gradient elasticity. As already fully discussed, one has to introduce an appropriate functional space to supply the most suitable space of configurations, where he has to find the solution of the problem of equilibrium under suitably applied external loads and imposed essential boundary conditions. In this paper, we recognize that such a naturally appropriate space is $\mathbf{H}_{1}(\operatorname{div}, V)$.

The presented results start from those already obtained for smooth domains: in fact, the well-posedness of the equilibrium problem was already proven in [20]. In order to give a solid mathematical basis to the related concepts of edge line contact forces and surface double forces, in the present paper we considered for continua reference configurations a particular, but rather interesting, class of non-smooth domains: i.e. polyhedral Lipschitz domains with edges. We have proved that the presence of edges produces the appearance of contact line forces acting on them. Moreover, we prove that in the presence of externally applied line forces on curves included in the faces of the boundary the surface contact double forces must undergo a discontinuity jump. The presented analysis treats the linearized elastic deformation only: however, the conditions (18)-(22) indicate that an interesting and important problem is opened by the obtained results. In fact, one must conjecture now that, in the theory of finite deformations the application of line forces on faces of the boundary of second gradient continua may produce, in the actual configuration, new edges. As a consequence, the space of configurations, in this case, must include placements that are not diffeomorphisms.

The interest of the study of the situations in which both reference or actual configurations can be polyhedral domains is therefore manifold. Together with the just mentioned considerations, one has to recall, first of all, that the geometrical singularities in the continua configurations, as edges and vertices, may essentially change the smoothness of solutions (see [49,50]). On the other hand, the analysis of solutions for polyhedral domains is important also in the context of the study of the most appropriate numerical methods to be applied to get the needed predictions by using introduced continuum models. Indeed, polygonal and polyhedral approximations are widely used within FEA and it is important to understand how this kind of approximations can influence the formulation of well-posed problems.

In conclusion, we can state that in this paper:

(i) the existence and uniqueness of weak solutions for the equilibrium problem of dilatational linear second gradient continua could be proven when the configuration space is a functional energy space whose norm is equivalent to the norm in $\mathbf{H}_{1}(\operatorname{div}, V)$;

(ii) the consequent well-posedness conditions for external loadings expressed in terms of Lebesgue and Sobolev's spaces were obtained; and

(iii) for what concerns in particular external double forces, we did manage to relax the previous requirements of [20] in such a way that these external loadings consists of piecewise differentiable generalized functions.

Our future research project concerns the study of the conditions in which, for finite deformations, one can observe the onset of new edges in the actual configuration.

\section{Funding}

The author(s) disclosed receipt of the following financial support for the research, authorship, and/or publication of this article: This work was supported by the Russian Science Foundation (grant number 20-41-04404, issued to the Institute of Applied Mechanics of Russian Academy of Sciences).

\section{ORCID iDs}

Victor A. Eremeyev iD https://orcid.org/0000-0002-8128-3262

Francesco dell'Isola (iD https://orcid.org/0000-0002-3607-1113

\section{References}

[1] Cordero, NM, Forest, S, and Busso, EP. Second strain gradient elasticity of nano-objects. J Mech Phys Solids 2016; 97 : 92-124.

[2] Askes, H, and Aifantis, EC. Gradient elasticity in statics and dynamics: An overview of formulations, length scale identification procedures, finite element implementations and new results. Int J Solids Struct 2011; 48(13): 1962-1990.

[3] Khakalo, S, and Niiranen, J. Form II of Mindlin's second strain gradient theory of elasticity with a simplification: For materials and structures from nano-to macro-scales. Eur J Mech A Solids 2018; 71: 292-319. 
[4] Abdoul-Anziz, H, and Seppecher, P. Strain gradient and generalized continua obtained by homogenizing frame lattices. Math Mech Complex Syst 2018; 6(3): 213-250.

[5] dell'Isola, F, and Steigmann, D. A two-dimensional gradient-elasticity theory for woven fabrics. J Elasticity 2015; 118(1): 113125.

[6] Rahali, Y, Giorgio, I, Ganghoffer, JF, et al. Homogenization à la Piola produces second gradient continuum models for linear pantographic lattices. Int J Eng Sci 2015; 97: 148-172.

[7] dell'Isola, F, and Steigmann, DJ. Discrete and Continuum Models for Complex Metamaterials. Cambridge: Cambridge University Press, 2020.

[8] dell'Isola, F, Seppecher, P, Alibert, JJ, et al. Pantographic metamaterials: An example of mathematically driven design and of its technological challenges. Continuum Mech Thermodyn 2019; 31(4): 851-884.

[9] dell'Isola, F, Seppecher, P, Spagnuolo, M, et al. Advances in pantographic structures: Design, manufacturing, models, experiments and image analyses. Continuum Mech Thermodyn 2019; 31(4): 1231-1282.

[10] dell'Isola, F, Lekszycki, T, Pawlikowski, M, et al. Designing a light fabric metamaterial being highly macroscopically tough under directional extension: First experimental evidence. Z angew Math Phys 2015; 66(6): 3473-3498.

[11] Eremeyev, VA, dell'Isola, F, Boutin, C, et al. Linear pantographic sheets: Existence and uniqueness of weak solutions. $J$ Elasticity 2018; 132(2): 175-196.

[12] Toupin, RA. Elastic materials with couple-stresses. Arch Rat Mech Anal 1962; 11(1): 385-414.

[13] Toupin, RA. Theories of elasticity with couple-stress. Arch Rat Mech Anal 1964; 17(2): 85-112.

[14] Mindlin, RD. Micro-structure in linear elasticity. Arch Rat Mech Anal 1964; 16(1): 51-78.

[15] Mindlin, RD, and Eshel, NN. On first strain-gradient theories in linear elasticity. Int J Solids Struct 1968; 4(1): $109-124$.

[16] Aifantis, EC. Update on a class of gradient theories. Mech Mater 2003; 35(3): 259-280.

[17] Yang, FACM, Chong, ACM, Lam, DCC, et al. Couple stress based strain gradient theory for elasticity. Int J Solids Struct 2002; 39(10): 2731-2743.

[18] Zhou, S, Li, A, and Wang, B. A reformulation of constitutive relations in the strain gradient elasticity theory for isotropic materials. Int J Solids Struct 2016; 80: 28-37.

[19] Sulem, J, and Vardoulakis, IG. Bifurcation Analysis in Geomechanics. Boca Raton, FL: CRC Press, 1995.

[20] Eremeyev, VA, Lurie, SA, Solyaev, YO, et al. On the well-posedness of static boundary value problem within the linear dilatational strain gradient elasticity. $Z$ angew Math Phys 2020; 71(6): 1-16.

[21] Eremeyev, VA, Cazzani, A, and dell'Isola, F. On nonlinear dilatational strain gradient elasticity. Continuum Mech Thermodyn 2021; DOI: 10.1007/s00161-021-00993-6.

[22] Lurie, SA, Kalamkarov, AL, Solyaev, YO, et al. Dilatation gradient elasticity theory. Eur J Mech A Solids 2021; 88: 104258.

[23] Nunziato, JW, and Cowin, SC. A nonlinear theory of elastic materials with voids. Arch Rat Mech Anal 1979; 72(2): $175-201$.

[24] Cowin, SC, and Nunziato, JW. Linear elastic materials with voids. J Elasticity 1983; 13(2): 125-147.

[25] Eringen, AC. Microcontinuum Field Theory. I. Foundations and Solids. New York: Springer, 1999.

[26] Korteweg, DJ. Sur la forme que prennent les équations des mouvements des fluides si l'on tient compte des forces capillaires par des variations de densité. Arch Néerland Sci Exactes Natur 1901; Sér. II(6): 1-24.

[27] Cahn, JW, and Hilliard, JE. Free energy of a nonuniform system. III. Nucleation in a two-component incompressible fluid. $J$ Chem Phys 1959; 31(3): 688-699.

[28] Seppecher, P. Second-gradient theory: Application to Cahn-Hilliard fluids. In Continuum Thermomechanics. Berlin: Springer, 2000, pp. 379-388.

[29] Seppecher, P. Les Fluides de Cahn-Hilliard. Mémoire d'habilitation à diriger des recherches, Université du Sud Toulon, 1996.

[30] Mareno, A, and Healey, TJ. Global continuation in second-gradient nonlinear elasticity. SIAM J Math Anal 2006; $38(1)$ : $103-115$.

[31] Healey, TJ, and Krömer, S. Injective weak solutions in second-gradient nonlinear elasticity. ESAIM Control Opt Calc Variat 2009; 15(4): 863-871.

[32] Germain, P. The method of virtual power in the mechanics of continuous media, I: Second-gradient theory. Math Mech Complex Syst 2020; 8(2): 153-190.

[33] Germain, P. Functional concepts in continuum mechanics. Meccanica 1998; 33(5): 433-444.

[34] Sedov, LI. Mathematical methods for constructing new models of continuous media. Russ Math Surv 1965; 20 (5): 123.

[35] Sedov, LI. Models of continuous media with internal degrees of freedom: PMM vol. 32, n 5, 1968, pp. 771-785. J Appl Math Mech 1968; 32(5): 803-819.

[36] Sedov, LI. Variational methods of constructing models of continuous media. In: Parkus, H and Sedov, LI (eds.) Irreversible Aspects of Continuum Mechanics and Transfer of Physical Characteristics in Moving Fluids. Vienna: Springer, pp. 346-358.

[37] Epstein, M, and Smelser, R. An appreciation and discussion of Paul Germain's "The method of virtual power in the mechanics of continuous media, I: Second-gradient theory". Math Mech Complex Syst 2020; 8(2): 191-199.

[38] dell'Isola, F, Della Corte, A, and Giorgio, I. Higher-gradient continua: The legacy of Piola, Mindlin, Sedov and Toupin and some future research perspectives. Math Mech Solids 2017; 22(4): 852-872.

[39] Abali, BE, Müller, WH, and dell'Isola F. Theory and computation of higher gradient elasticity theories based on action principles. Arch Appl Mech 2017; 87(9): 1495-1510.

[40] Eremeyev, VA, and Altenbach, H. Equilibrium of a second-gradient fluid and an elastic solid with surface stresses. Meccanica 2014; 49(11): 2635-2643. 
[41] Auffray, N, dell'Isola, F, Eremeyev, VA, et al. Analytical continuum mechanics à la Hamilton-Piola least action principle for second gradient continua and capillary fluids. Math Mech Solids 2015; 20(4): 375-417.

[42] Eremeyev, VA, Cloud, MJ, and Lebedev, LP. Applications of Tensor Analysis in Continuum Mechanics. Hackensack, NJ: World Scientific, 2018.

[43] Bertram, A, and Forest, S (eds.) Mechanics of Strain Gradient Materials. Cham: Springer International Publishing, 2020.

[44] Lions, JL, and Magenes, E. Non-Homogeneous Boundary Value Problems and Applications, Vol. 1. (Die Grundlehren der mathematischen Wissenschaften, Vol. 181). Berlin: Springer, 1972.

[45] Fichera, G. Existence theorems in elasticity. In: Flügge S (ed.) Handbuch der Physik, Vol. VIa/2. Berlin: Springer, 1972, pp. 347-389.

[46] Ladyzhenskaya, A. The Boundary Value Problems of Mathematical Physics (Applied Mathematical Sciences, Vol. 49). New York: Springer, 1985.

[47] Girault, V, and Raviart, PA. Finite Element Methods for Navier-Stokes Equations: Theory and Algorithms. Berlin: Springer, 1986.

[48] Lebedev, LP, Vorovich, II, and Cloud, MJ. Functional Analysis in Mechanics. 2nd ed. New York: Springer, 2013.

[49] Maz’ja, VG. Sobolev Spaces (Springer Series in Soviet Mathematics). Berlin: Springer, 1985.

[50] Grisvard, P. Elliptic Problems in Nonsmooth Domains. Boston, MA: Pitman, 1985.

[51] Guo, B, and Babuška, I. Regularity of the solutions for elliptic problems on nonsmooth domains in $r^{3}$, Part I: countably normed spaces on polyhedral domains. Proc R Soc Edinb Sec A Math 1997; 127(1): 77-125.

[52] Guo, B, and Babuška, I. Regularity of the solutions for elliptic problems on nonsmooth domains in $\mathbb{R}^{3}$. Part II: Regularity in neighbourhoods of edges. Proc R Soc Edinb Sec A Math 1997; 127(3): 517-545.

[53] Geymonat, G. Trace theorems for Sobolev spaces on Lipschitz domains. Necessary conditions. Ann Math Blaise Pascal 2007; 14(2): 187-197.

[54] Buffa, A, and Geymonat, G. On traces of functions in $w^{2, p}(\omega)$ for Lipschitz domains in $\mathbb{R}^{3}$. C R Acad Sci Ser I Math 2001; 332(8): 699-704.

[55] Geymonat, G, and Krasucki, F. On the existence of the Airy function in Lipschitz domains. Application to the traces of $h^{2}$. $C R$ Acad Sci Ser I Math 2000; 330(5): 355-360.

[56] Adams, RA, and Fournier, JJF. Sobolev Spaces (Pure and Applied Mathematics, Vol. 140). 2nd ed. Amsterdam: Academic Press, 2003.

[57] Fichera, G. Linear Elliptic Differential Systems and Eigenvalue Problems (Lecture Notes in Mathematics, Vol. 8). Berlin: Springer, 1965.

[58] Agranovich, M. Elliptic boundary problems. In: Agranovich, M, Egorov, Y, and Shubin, M (eds.) Partial Differential Equations IX: Elliptic Boundary Problems (Encyclopaedia of Mathematical Sciences, Vol. 79). Berlin: Springer, pp. 1-144.

[59] Hörmander, L. The Analysis of Linear Partial Differential Operators. II. Differential Operators with Constant Coefficients (A Series of Comprehensive Studies in Mathematics, Vol. 257). Berlin: Springer, 1983.

[60] Placidi, L, Barchiesi, E, Turco, E, et al. A review on 2D models for the description of pantographic fabrics. Z angew Math Phys 2016; 67(5): 121.

[61] Eremeyev, VA, Alzahrani, FS, Cazzani, A, et al. On existence and uniqueness of weak solutions for linear pantographic beam lattices models. Continuum Mech Thermodyn 2019; 31(6): 1843-1861.

[62] Eremeyev, VA, and dell'Isola, F. Weak solutions within the gradient-incomplete strain-gradient elasticity. Lobachevskii J Math 2020; 41(10): 1992-1998.

[63] Lurie, AI. Theory of elasticity. In: Foundations of Engineering Mechanics. Berlin: Springer, 2005.

[64] Buffa, A, and Ciarlet P, Jr. On traces for functional spaces related to Maxwell's equations. Part I: An integration by parts formula in Lipschitz polyhedra. Math Meth Appl Sci 2001; 24(1): 9-30.

[65] Wilson, EB. Vector Analysis, Founded upon the Lectures of G. W. Gibbs. New Haven, CT: Yale University Press, 1901.

[66] Tartar, L. An Introduction to Sobolev Spaces and Interpolation Spaces. Berlin: Springer, 2007.

[67] Buffa, A, and Ciarlet, P Jr. On traces for functional spaces related to Maxwell's equations Part II: Hodge decompositions on the boundary of Lipschitz polyhedra and applications. Math Meth Appl Sci 2001; 24(1): 31-48.

[68] Saks, RS. Operator Vdiv and Sobolev spaces (in Russian). Dinamicheskie Sistemy 2018; 8(4): 385-407.

[69] Saks, RS. The gradient-of-divergence operator in $l_{2}(g)$. Dokl Math 2015; 91(3): 359-363.

[70] Le Quang, H, and He, QC. The number and types of all possible rotational symmetries for flexoelectric tensors. Proc R Soc A Math Phys Eng Sci 2011; 467(2132): 2369-2386.

[71] Olive, M, and Auffray, N. Symmetry classes for odd-order tensors. ZAMM 2014; 94(5): 421-447.

[72] dell'Isola, F, and Seppecher, P. Edge contact forces and quasi-balanced power. Meccanica 1997; 32(1): 33-52.

[73] dell'Isola, F, Seppecher, P, and Madeo, A. How contact interactions may depend on the shape of Cauchy cuts in $n$th gradient continua: approach “á la d'Alembert”. Z angew Math Phys 2012; 63(6): 1119-1141.

[74] Eremeyev, VA, and Lebedev, LP. Existence of weak solutions in elasticity. Math Mech Solids 2013; $18(2): 204-217$.

[75] Capriz, G. Continua with Microstructure. New York: Springer, 1989. 